\title{
Space Plasma Exploration by Active Radar (SPEAR): an overview of a future radar facility
}

\author{
D. M. Wright ${ }^{1}$, J. A. Davies ${ }^{1}$, T. R. Robinson ${ }^{1}$, P. J. Chapman ${ }^{1}$, T. K. Yeoman ${ }^{1}$, E. C. Thomas ${ }^{1}$, \\ M. Lester ${ }^{1}$, S. W. H. Cowley ${ }^{1}$, A. J. Stocker ${ }^{2}$, R. B. Horne ${ }^{3}$, F. Honary ${ }^{4}$ \\ ${ }^{1}$ Department of Physics \& Astronomy, University of Leicester, University Road, Leicester, LE1 7RH, UK \\ 2 Department of Engineering, University of Leicester, University Road, Leicester, LE1 7RH, UK \\ ${ }^{3}$ British Antarctic Survey, Madingley Road, Cambridge, CB3 0ET, UK \\ ${ }^{4}$ Department of Communication Systems, University of Lancaster, Bailrigg, Lancaster, LA1 4YR, UK
}

Received: 17 March 2000 / Revised: 10 May 2000 / Accepted: 23 May 2000

\begin{abstract}
SPEAR is a new polar cap HF radar facility which is to be deployed on Svalbard. The principal capabilities of SPEAR will include the generation of artificial plasma irregularities, operation as an 'all-sky' HF radar, the excitation of ULF waves, and remote sounding of the magnetosphere. Operation of SPEAR in conjunction with the multitude of other instruments on Svalbard, including the EISCAT Svalbard radar, and the overlap of its extensive field-of-view with that of several of the HF radars in the SuperDARN network, will enable in-depth diagnosis of many geophysical and plasma phenomena associated with the cusp region and the substorm expansion phase. Moreover, its ability to produce artificial radar aurora will provide a means for the other instruments to undertake polar cap plasma physics experiments in a controlled manner. Another potential use of the facility is in 'field-line tagging' experiments, for coordinated ground-satellite experiments. Here the scientific objectives of SPEAR are detailed, along with the proposed technical specifications of the system.
\end{abstract}

Key words: Ionosphere (active experiments) - Radio science (instruments and techniques) - Space plasma physics (instruments and techniques)

\section{Introduction}

Increasingly, it is realized that further improvements in our understanding of the field of solar-terrestrial physics are only likely to result from better coordinated observing programmes involving a number

Correspondence to: D. M. Wright e-mail: Darren.Wright@ion.le.ac.uk of complementary diagnostics, reliant not least upon improved coordination between ground- and satellite-based observations. SPEAR (Space Plasma Exploration by Active Radar) is a new concept in ground-based radar design, intended to take this complementarity a stage further. SPEAR will combine a HF coherent scatter radar capability with that of an active system, the latter enabling the artificial generation of VLF and ULF waves as well as short-scale plasma density irregularities. The facility is to be deployed on Spitzbergen, an island in the Svalbard archipelago, some one thousand kilometers north of mainland Norway. The latitude of Svalbard places the SPEAR facility, in general, on open magnetic field lines. This location is also in the vicinity of the distinctive cusp region of the dayside auroral zone, which although on open field lines is near the openclosed field line boundary. On the nightside the radar will be ideally situated to diagnose the dynamics of the expanding auroral bulge associated with the substorm expansion phase onset. Svalbard and northern Scandinavia are already host to a wide variety of instrumentation, not least the EISCAT mainland sites and the EISCAT Svalbard radar (ESR). There is substantial overlap of the proposed field-of-view of SPEAR with that of several of the HF radars in the SuperDARN network of coherent scatter radars (e.g. Greenwald et al., 1995), including both the Finland and Iceland East CUTLASS radars. The active capabilities of the SPEAR radar will greatly enhance operation of such facilities, which rely at present on the occurrence of natural plasma irregularities. These and the other scientific objectives of the SPEAR facility are described in more detail below. Figure 1 presents a map illustrating the location of Svalbard and SPEAR with respect to the CUTLASS fields-ofview, the EISCAT sites, and magnetometer networks. The currently scheduled deployment of SPEAR during the second half of 2002 will permit full operation of the facility to be commenced early the following year. 


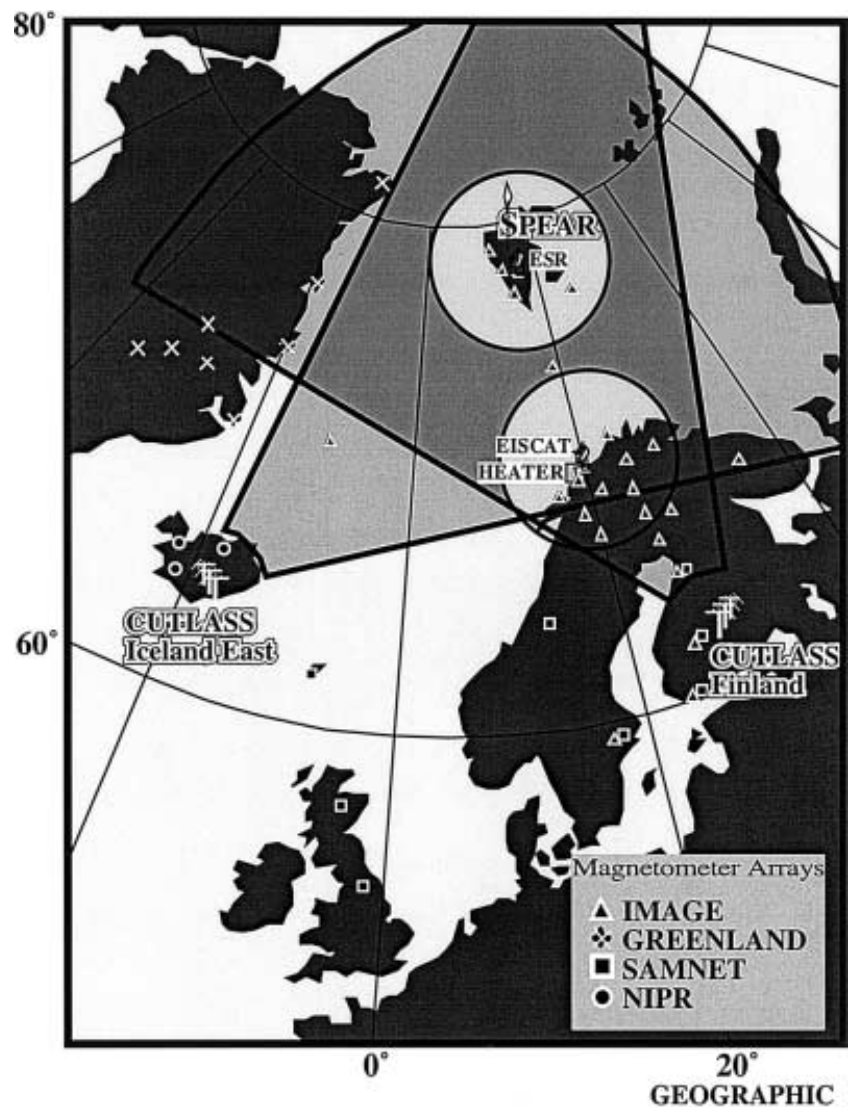

Fig. 1. An illustration of the location of Svalbard and SPEAR with respect to the CUTLASS fields of view and nearby magnetometer networks

\section{Scientific objectives}

\subsection{The generation of artificial field aligned irregularities}

Coherent radars, such as those of the SuperDARN network, play a vital role in imaging high-latitude dynamical processes by mapping plasma convection orthogonal to the geomagnetic field, on a wide variety of spatial and temporal scales. However, their operation is limited to times when small-scale plasma irregularities are present. Moderately high-power radio waves, those of some $10 \mathrm{MW}$ effective radiated power (ERP), can readily generate small-scale irregularities in both the Eand F-regions under a wide variety of conditions, as has been shown using the EISCAT heating facility at Tromsø (e.g. Robinson, 1989). Such artificially generated irregularities can act as targets for coherent scatter radars; this has been demonstrated on a number of occasions using the Finland and Iceland East radars of the CUTLASS facility, coverage of both of which includes the Tromsø heater (e.g. Bond et al., 1997; Robinson et al., 1997, 1998). This situation is illustrated schematically in Fig. 2a. Artificially produced irregularities have been found to behave just as those occurring naturally with regard to yielding information about convection flow velocities (Eglitis et al., 1998). The occurrence of the artificial CUTLASS backscatter can also provide a platform for high spatial and temporal resolution measurements of naturally occurring geomagnetic pulsations (e.g. Yeoman et al., 1997; Wright and Yeoman, 1999). Figure 3 presents data from an experiment of this type on 15 October, 1998, during which both the CUTLASS Finland and Iceland East radars were making measurements in a high spatial and temporal resolution mode, $15 \mathrm{~km}$ and $10 \mathrm{~s}$, respectively (Wright and Yeoman, 1999). Figure 3a, b illustrates time series of irregularity drift velocity from Finland and Iceland at various ranges through the region of heatergenerated scatter. The signatures of the ULF waves, modulating the background ionospheric flow, are clearly visible in the data. Without the benefit of artificially generated irregularities the pulsations would have been completely invisible to CUTLASS. The drift velocities, observed in a volume common to both radar fields of view, were merged and resolved into north-south and east-west components (Fig. 3c, d). Since the spectral widths associated with the artificial scatter are very narrow (typically less than $10 \mathrm{~m} \mathrm{~s}^{-1}$ ), the data in these panels represent high (15 km spatial and $10 \mathrm{~s}$ temporal) resolution measurements of the electric fields associated with ULF waves, at an accuracy far higher than previously achieved by any instrument in solar-terrestrial physics. This demonstrates the power of artificial modification as a tool for diagnosing natural phenomena in the ionosphere. It is anticipated that SPEAR will significantly extend the operational capabilities of CUTLASS over Svalbard, which will be particularly valuable when combined with coordinated experiments with the ESR, especially those involving satellite conjunctions.

\subsection{ULF modulation for magnetospheric sounding}

A wide variety of ULF and VLF oscillations can be stimulated by modulated heating of the high latitude ionospheric electrical current system (e.g. Stubbe et al., 1982). The collisional heating of electrons in the ionospheric D- and E-regions alters the temperaturedependent conductivity, which results in changes in the natural current flow. Under modulated heating, the heated region, in effect, behaves as a virtual antenna radiating waves at the modulation frequency. The relatively low number of reports of successful incidences of this type of experiment is mainly due to the fact that the optimum conditions required for success occur infrequently. However, the artificial stimulation of ULF waves has been achieved on a number of occasions. Previous long period modulation experiments with the Tromsø heater have produced magnetic field amplitudes of up to $14 \mathrm{nT}$ on the ground (e.g. Stubbe and Kopka, 1981; Stubbe et al., 1982; Stubbe, 1996). Spitzbergen is located such that the dayside polar electrojet will commonly occur above the SPEAR facility. In addition, substorm associated electrojets reach Svalbard not infrequently. On the occasions when this current system can be heated, modulating the 


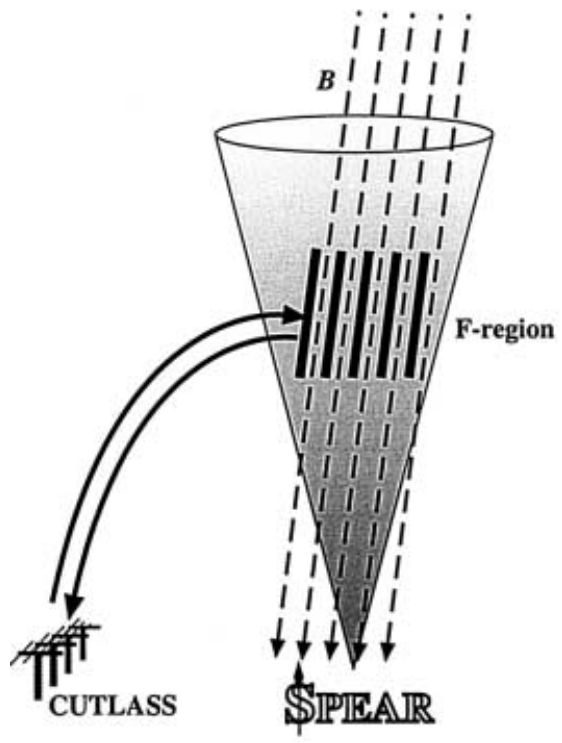

a) Generation of artificial field aligned irregularities

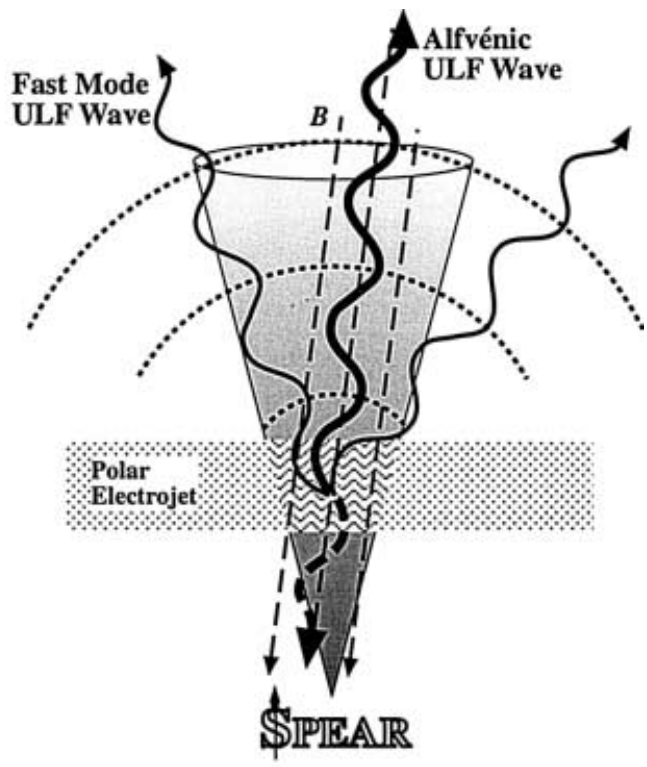

b) Stimulation of ULF waves
Fig. 2. Schematics representing a the generation of artificial field aligned irregularities by SPEAR utilising its high power transmitters and $\mathbf{b}$ the excitation of Alfvénic (resonant) ULF waves, which form a standing mode along magnetic field lines, and fast-mode ULF (non-resonant) waves currents flowing in the polar ionosphere with SPEAR will allow experimental studies into the importance of magnetosphere-ionosphere coupling. For example, it will be possible to actively test theories of quiet auroral arc formation (e.g. Lysak, 1991). SPEAR will allow the ionospheric conductivity to be modified in a systematic and controlled manner so that the causal relationships involved can be quantified. Furthermore, such periodic modulation of ionospheric conductivities introduces the possibility of using the resonance to drive ULF standing waves of significant amplitudes (see Fig. 2b), the periods of which are good indicators of characteristic scale sizes in the magnetosphere. Interhemispheric studies of these resonances may also be possible since the geomagnetically conjugate location of SPEAR will lie within the fields of view of the SuperDARN Kerguelen and Syowa East radars. More importantly, with the combination of SPEAR in the polar cap and the Tromsø heater in the auroral zone it will also be possible to investigate the open/closed field line boundary of the magnetosphere, since the response from the open field lines to modulated heating in the cap should be noticeably different from that in the closed field line region.

\subsection{Injection of field-guided waves}

Much of the coupling and communication between different parts of the space plasma system is along magnetic field lines, which are themselves highly dynamic. In coordinated ground-satellite observations, vital in the study of such important issues as the identification of the mechanisms and locations associated with reconnection and substorm triggering, it is necessary to identify common field lines. Using an active high-power radar, field lines can be 'tagged' with injected waves (and possibly particles accelerated by artificially stimulated electrostatic waves) which can themselves be detected by satellite-born instruments (see Fig. 4a). It was originally suggested that this might be achieved with heater-stimulated VLF waves with frequencies of a few $\mathrm{kHz}$. Indeed, VLF waves, generated by the heater at Tromsø, were observed as far out as $11000 \mathrm{~km}$ by the DE-1 satellite (James et al., 1990). Ray tracing studies carried out as part of a preliminary feasibility study for SPEAR indicate that these waves excited by modulated electrojet heating are not sufficiently well guided to be detectable by spacecraft on a common field line. However, the guiding efficiency of the magnetic field increases as the wave frequency is reduced such that waves with frequencies of a few $\mathrm{Hz}$ appear to be trapped on field lines out to at least 4 or 5 Earth radii. This is demonstrated in Fig. 5, which illustrates the path of a $3 \mathrm{~Hz}$ ULF wave injected from the ionosphere over Svalbard, and is an extremely encouraging result, providing sufficient evidence that this ambitious experiment is worth trying with appropriate spacecraft, such as Cluster II. The Cluster system with its multi-satellite geometry is ideal for testing SPEAR's wave injection capabilities, since there is far more chance of an encounter with a tagged field line with a distribution of satellite-born instruments. Since it is possible to modulate ionospheric currents with a superposed set of frequencies it would be sensible to transmit waves in, say, three frequency bands simultaneously (e.g. $1 \mathrm{~Hz}, 30 \mathrm{~Hz}, 1000 \mathrm{~Hz}$ ), which would diverge or leak from the field line by different amounts. A spacecraft traversing the region would encounter something like an archery target of spreading waves. The lower the frequency its wave instruments were able to detect the closer the spacecraft would be to the tagged field line. Ideally, both SPEAR and the Tromsø heater 


\section{SUPERDARN PARAMETER PLOT CUTLASS: Finland and Iceland East (vel)}

\section{Oct 1998}

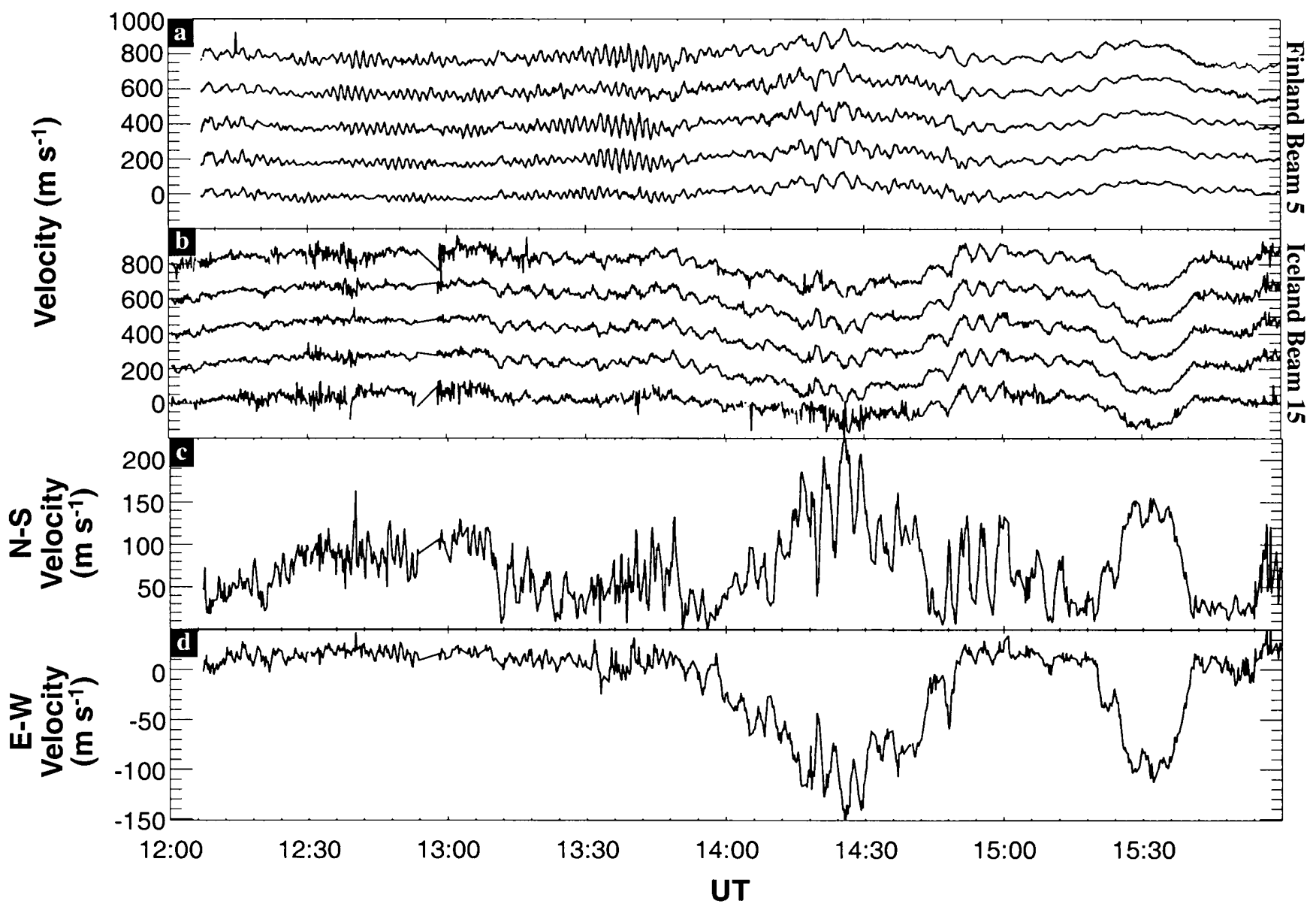

Fig. 3a-d. CUTLASS Finland and Iceland velocity measurements from a Tromsø heating experiment on 15 October 1998. a, b Illustrates time series of irregularity drift velocity from Finland and Iceland, respectively, at various ranges through the region of heater-

generated scatter. c, d Presents the north-south and east-west components of the field-orthogonal drift velocity, derived from the merged velocities in the region of the field-of-view common to both radars

could be operated simultaneously with slightly different frequency sets. This will allow the location of satellites to be determined in magnetic coordinates over an extended region in this important polar cap/auroral oval boundary region.

\subsection{All-sky HF backscatter from short-scale field aligned plasma density irregularities}

Because of the extremely high magnetic dip at Svalbard $\left(\sim 84^{\circ}\right)$, field aligned plasma irregularities, which coherently backscatter radar signals incident orthogonal to the geomagnetic field, lie almost vertically with respect to the ground plane. This, combined with the refraction provided by the ionosphere at appropriately chosen HF frequencies, means that backscatter is easily achieved at all azimuths (see Fig. 4b). This capability, which has a relatively low power requirement, will provide radial line-of-sight velocities with $15 \mathrm{~km}$ range resolution in a scattering volume with a horizontal cross-section corresponding to a roughly circular region with a radius of several hundred kilometres (depending on propagation conditions), centred on the SPEAR site. Figure 6 displays a ray-trace of the expected field of view of SPEAR. The vertical extent of the potential scattering region will extend from $100 \mathrm{~km}$ to $400 \mathrm{~km}$ in the ionosphere. This extensive scattering volume will also provide excellent opportunities for exploring the development, structure and dynamics of field aligned plasma irregularities which play a major role in the electrodynamics and thermodynamics of the high latitude ionosphere. This configuration is also intended to explore the possibility of obtaining coherent scatter from waves and irregularities in the magnetosphere, under underdense propagation conditions when the radar waves penetrate the F-region. Such long-range scatter has occasionally been reported in the literature (Hysell et al., 1997), but has not yet been studied systematically. Such scatter could provide important new information concerning 


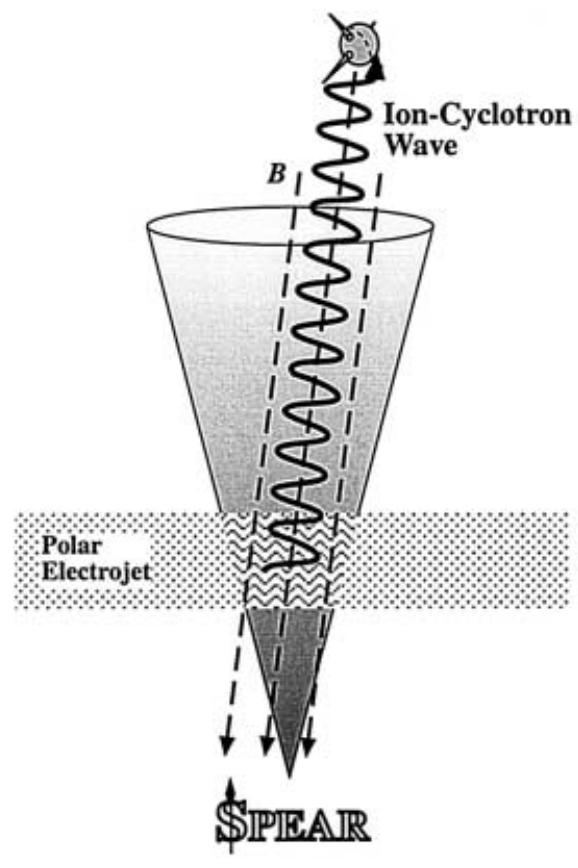

a) Field guided wave injection

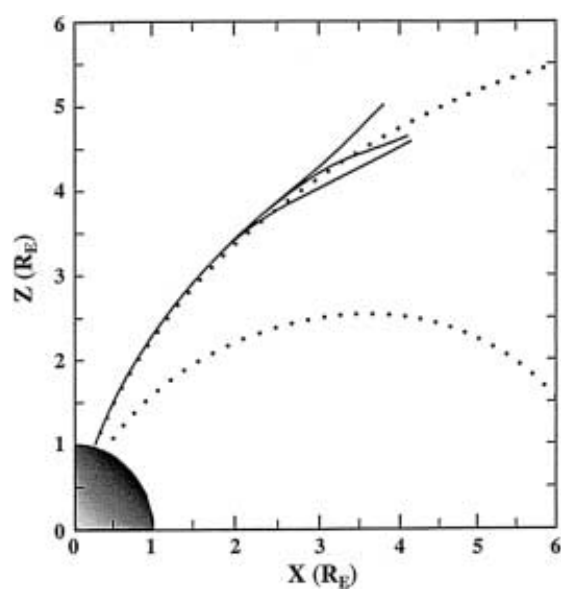

Fig. 5. A raytrace of the path of a $3 \mathrm{~Hz}$ field guided ULF wave injected from the ionosphere above SPEAR (see Fig. 4a). It is suggested that such a low frequency wave would remain field guided to altitudes high enough for it to be detected by spacecraft, thus marking a narrow flux tube in space

narrow regions of field aligned currents and the instabilities and waves they generate in the magnetosphere.

\subsection{Nonlinear interactions in the polar cap ionosphere}

High-power HF electromagnetic waves can excite a number of instabilities in the ionosphere, as is evidenced by the wealth of results from the EISCAT heating experiment (e.g. Robinson, 1989; Robinson et al., 1997, 1998; Bond et al., 1997; Stubbe, 1996). The location of a new active radar on Svalbard will provide an opportu- nity to undertake studies into the nonlinear interaction of radio waves with the unique space plasma regime which exists in the polar cap. In particular, the cusp region has unique particle precipitation characteristics (in terms of energy distributions and access to special magnetic field line geometries) which will allow waveparticle interaction studies to be undertaken for the first time in this environment.

\section{Technical specifications}

Experience with the high-power HF system at Tromsø gives us a reasonable idea of what power levels are required to achieve most of the capabilities outlined already. In particular, experiments with the Tromsø heater have demonstrated that artificial radar aurora can be produced at power levels of as low as $10 \mathrm{MW}$ of ERP. The ionospheric scatter for the all-sky radar also has a low power requirement; indeed for such operation SPEAR would transmit an ERP comparable to that of the existing SuperDARN radars, of 0.4 MW. However, artificial ULF sounding, field guided wave injection, and nonlinear interactions need powers of several times that level. With this in mind, a phased approach has been adopted for construction and deployment of SPEAR which will allow the system capabilities to evolve. A modular system of distributed transmitters is envisaged as the best approach. This allows the construction of a system to deliver 28 MW ERP, with fewer antennas and transmitters than are required for the full system. An ERP of $28 \mathrm{MW}$ would be sufficient to generate artificial radar aurora and permit operation of SPEAR as an 


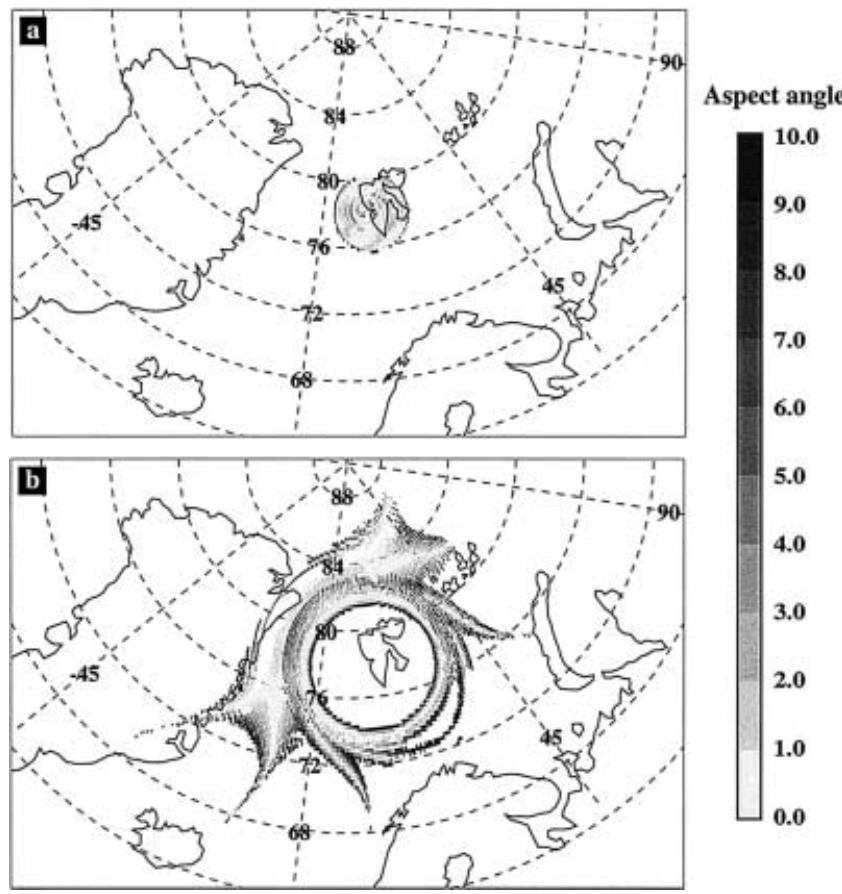

Fig. 6a, b. Angle between the k-vector and the Earth's magnetic field as a function of position derived from a ray-tracing simulation for a $4 \mathrm{MHz}$ signal transmitted from the proposed SPEAR transmitter during a day time $\left(f_{\mathrm{OF}} 2=5.8 \mathrm{MHz}\right)$ and $\mathbf{b}$ night time $\left(f_{\mathrm{oF}} 2=\right.$ 3.2 $\mathrm{MHz})$

all-sky radar. This is currently the limit of the funding for the system. It is hoped that funding from international collaborators will facilitate the realisation of a second stage of SPEAR which would bring the ERP up to $68 \mathrm{MW}$ which will then provide the remaining capabilities. The phasing of construction work will have the advantage of spreading antenna field construction over two summers, which is an important consideration in a hostile climate such as that on Svalbard. The modular approach also provides sufficient flexibility for any future evolution of the system. The phased development, in addition, enables system performance to be checked and validated in stages. However, even after opting for this modular approach there still remains a variety of possible configuration options.

Currently the proposed configuration for SPEAR is based on a $6 \times 4$ array of rhombically broadened full wave crossed dipole antennas (similar to those utilised in the Tromsø heating facility; see Fig. 7), each dipole being driven by a $4 \mathrm{~kW}$ solid state transmitter designed specifically for SPEAR. The system will operate in the frequency range 4-6 MHz and the total ERP from all 48 transmitters will be $28 \mathrm{MW}$. For comparison, this is about $10 \mathrm{~dB}$ lower than the output of the EISCAT heater at Tromsø. This distributed transmitter configuration is similar to that employed by the HAARP (High frequency Active Auroral Research Program; Rodriguez et al., 1998) high-power facility located in Gakona, Alaska, USA. In order for SPEAR to operate in HF radar mode, a solid state high power transmit/receive antenna switch had to be designed and developed to

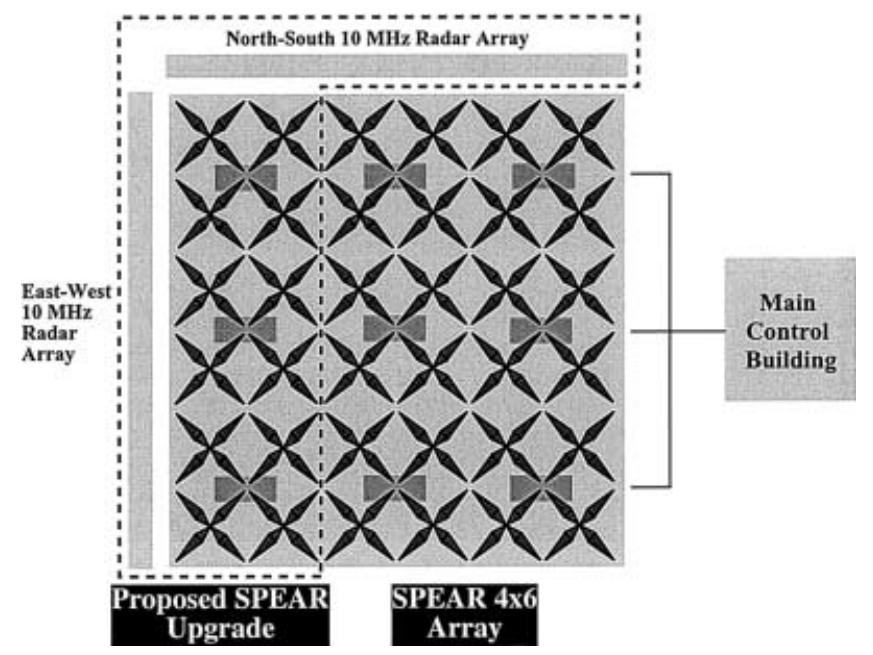

Fig. 7. The proposed antenna/transmitter arrangement for SPEAR consists of a $4 \times 6$ array of crossed dipole antennas, each driven by its own solid state high power transmitter. Phasing of the signals will allow the beam to be steered north-south and east-west. The region inside the dashed line indicates a possible configuration for the proposed system upgrade, where the high power array is expanded to a $6 \times 6$ array and a new L-shaped array is introduced to improve the performance of the all-sky radar mode of operation

allow rapid switching. The antenna beam pattern of the system will be controllable to within $20^{\circ}$ off the zenith and over $360^{\circ}$ of azimuth. The zenith beam width (Fig. 8a, b) will be asymmetrical due to the dimensions of the array and will range from $21^{\circ} \times 14.2^{\circ}$ at $3.9 \mathrm{MHz}$ to $15.2^{\circ} \times 10.2^{\circ}$ at $5.6 \mathrm{MHz}$ with respective gains of $20.6 \mathrm{dBi}$ and $23.4 \mathrm{dBi}$ at these frequencies. Moreover, the array antennas will function as half wave dipoles facilitating the use of additional frequencies at half the designed full wave centre frequency.

Plans exist for a proposed upgrade of SPEAR assuming additional funding from international collaborators can be found. The upgrade will, if realised, involve the extension of the antenna array in two ways. The antenna arrangement will become a $6 \times 6$ array by the addition of extra antennas. During high power operations (generating artificial irregularities) the beam width will then become symmetrical and range from $14.2^{\circ}$ at $3.9 \mathrm{MHz}$ to $10.2^{\circ}$ at $5.6 \mathrm{MHz}$ (Fig. 8c, d). Secondly, the addition of new arrays will significantly improve the performance of SPEAR in HF radar mode. Although it must be stressed that the details of the HF radar configuration is not yet finalised, one possibility could be the addition of a new L-shaped array of either monopole or half wave dipole antennas at the perimeter of the $6 \times 6$ array (see Fig. 7), comprising of northsouth and east-west $2 \times 32$ element sub-arrays. This mode could operate at $10 \mathrm{MHz}$ with a beam at a fixed elevation angle. The new configuration would provide a radar beam $\sim 4^{\circ}$ wide in azimuth and $\sim 24^{\circ}$ wide in elevation (see Fig. 8e, f) and would, due to the selection of an optimum antenna separation, be steerable over most if not all azimuths.

The main time constraint on deployment is that imposed by the requirement to have SPEAR operational 

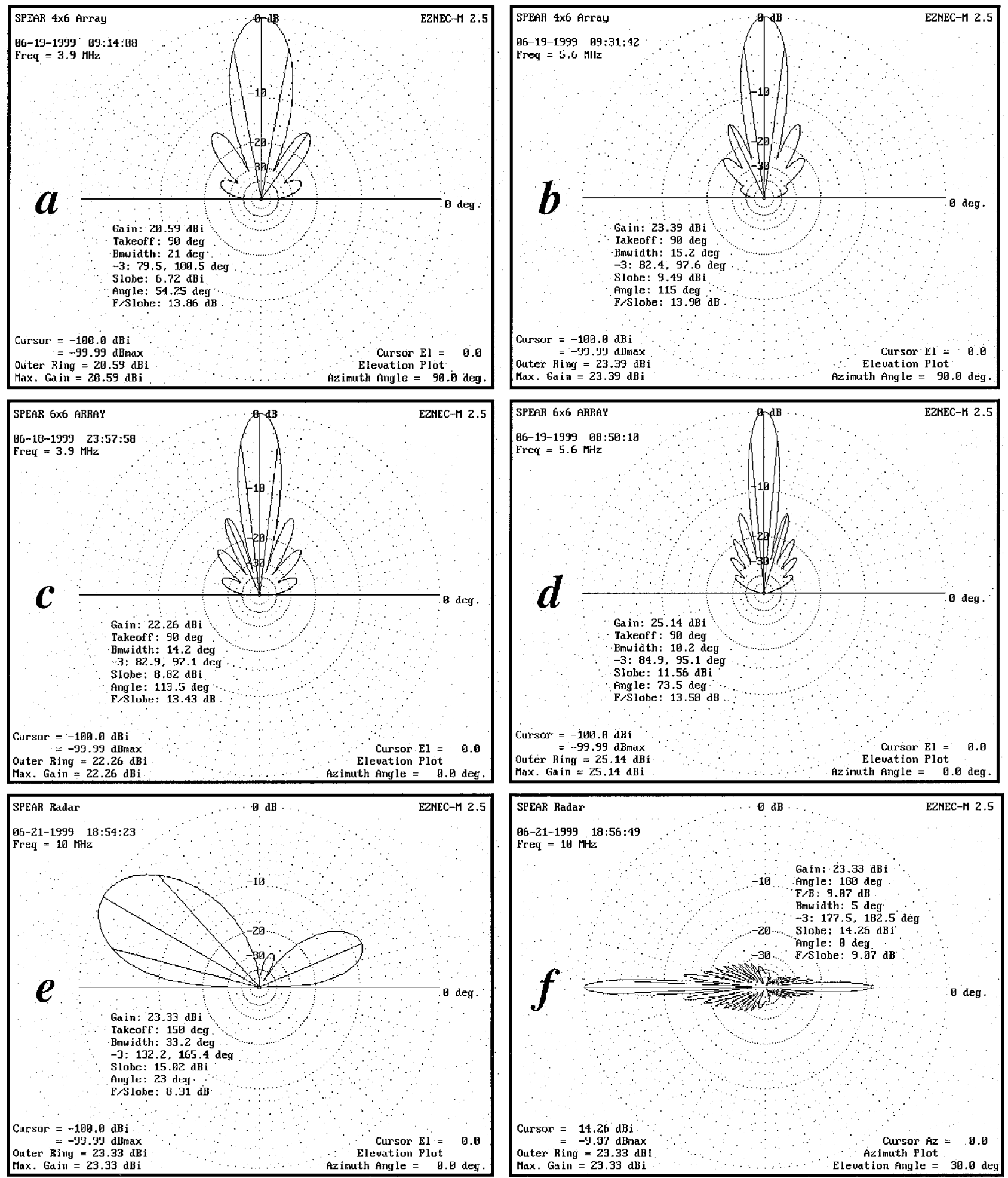

Fig. 8a-f. Polar diagrams of the expected radiation beam pattern from SPEAR for the $4 \times 6$ array operating at a $3.9 \mathrm{MHz}$ and b $5.6 \mathrm{MHz}$; a $6 \times 6$ array following the proposed system upgrade for $\mathbf{c}$

3.9 $\mathrm{MHz}$ and $\mathbf{d} 5.6 \mathrm{MHz}$; the all-sky HF radar mode utilising an Lshaped additional array (see text) viewed in e elevation and $\mathbf{f}$ azimuth

for a significant fraction of the Cluster operating period. It is likely, with these considerations in mind, that following the construction of an operational engineering model, comprising four crossed dipole

pairs driven by $8 \times 4 \mathrm{~kW}$ transmitters, by the end of June 2000, the remaining hardware will be built up to the end of 2001. The system will be deployed on Svalbard during 2002 and SPEAR will be operational 
in early 2003. The development of the proposed upgrade would occur subsequent to this, assuming additional funding could be secured from international collaborators.

\section{Summary}

An overview of the future SPEAR facility has been presented. With an ever increasing interest in the solarterrestrial physics of the polar/cusp region, the need for a system like SPEAR is clear. Not only will its standalone capabilities provide a means of solving some key issues of this region in the magnetosphere-ionosphere system but it will also complement existing and future instruments by offering the controlled excitation of ionospheric plasma instabilities, leading to the diagnosis of polar space plasma dynamics. SPEAR is a novel new concept in ground-based radar design incorporating the development of new and innovative technologies. SPEAR is now under construction and it is anticipated that this will be operational in early 2003. However, the proposed evolution of the system will require financial and scientific input from an international source. Such a collaborative partnership is welcomed at any time.

The capabilities of the facility will range from hightime resolution measurements of small scale plasma dynamics to the diagnosis of large-scale ionospheric plasma convection and magnetospheric structure and dynamics. It will complement other systems like ESR and CUTLASS, enhancing their capabilities and extending their coverage. The combination of SPEAR with the EISCAT UHF radars on the Norwegian mainland and the Tromsø heater, the ESR and CUTLASS, together with the Cluster satellites will provide a unique opportunity for coordinated observations of ionosphere-magnetosphere coupling processes across the important open/closed field line boundary between the polar cap and the auroral zone.

Acknowledgements. The authors would like to express their gratitude to Dr Mike Rietveld of the EISCAT scientific association for helpful technical discussions and to the Particle Physics and Astronomy Research Council (PPARC) for providing the funding necessary to develop and construct SPEAR.

Topical Editor G. Chanterer thanks a referee for his help in evaluating this paper.

\section{References}

Bond, G. E., T. R. Robinson, P. Eglitis, D. M. Wright, A. J. Stocker, M. T. Rietveld, and T. B. Jones, Decay characteristics of heater induced irregularities over Tromsø, Ann. Geophysicae, 15, 1412, 1997.
Eglitis, P., T. R. Robinson, M. T. Rietveld, D. M. Wright, and G. E. Bond, The phase speed of artificial irregularities observed by CUTLASS during HF modification of the auroral ionosphere, J. Geophys. Res., 103, 2253, 1998.

Greenwald, R. A., K. B. Baker, J. R. Dudeney, M. Pinnock, T. B. Jones, E. C. Thomas, J.-P. Villain, J.-C. Cerisier, C. Senior, C. Hanuise, R. D. Hunsucker, G. Sofko, J. Koehler, E. Nielsen, R. Pellinen, A. D. M. Walker, N. Sato and H. Yamagishi, DARN/SuperDARN: a global view of the dynamics of highlatitude convection, Space Sci. Rev., 71, 761, 1995.

Hysell, D. L., M. C. Kelley, A. V. Gurevich, A. N. Karashtin, A. M. Babichenko, Y. M. Yampolski, V. S. Beley, and J. F. Providakes, HF radar probing of the lower magnetosphere, J. Geophys. Res., 102, 4865, 1997.

James, H. G., U. S. Inan, and M. T. Rietveld, Observations on the DE-1 spacecraft of ELF/VLF waves generated by an ionospheric heater, J. Geophys. Res., 95, 12187, 1990.

Lysak, R. L., Feedback instability of the ionospheric resonant cavity, J. Geophys. Res., 96, 1553, 1991.

Robinson, T. R., The heating of the high-latitude ionosphere by high power radio waves, Phys. Rep., 179, 79, 1989.

Robinson, T. R., A. J. Stocker, G. E. Bond, P. Eglitis, D. M. Wright, and T. B. Jones, O- and X-mode heating effects observed simultaneously with the CUTLASS and EISCAT radars and low power HF diagnostics at Tromsø, Ann. Geophysicae, 15, 134, 1997.

Robinson, T. R., A. Stocker, G. Bond, P. Eglitis, D. Wright, T. B. Jones, and M. T. Rietveld, First CUTLASS-EISCAT heating results, Adv. Space. Res., 21, 663, 1998.

Rodriguez, P. E., J. Kennedy, M. J. Keskinen, C. L. Siefring, S. Basu, M. McCarrick, J. Preston, M. Engebretson, M. L. Kaiser, M. D. Desch, K. Goetz, J.-L. Bougeret, and R. Manning, The WIND-HAARP experiment: initial results of high power radio wave interactions with space plasmas, Geophys. Res. Lett., 25, 257, 1998.

Stubbe, P., Review of ionospheric modification experiments at Tromsø, J. Atmos. Terr. Phys., 58, 349, 1996.

Stubbe, P., and H. Kopka, Generation of Pc5 pulsations by polar electrojet modulation: first experimental evidence, J. Geophys. Res., 86, 1606, 1981.

Stubbe, P., H. Kopka, H. Lauche, M. T. Rietveld, A. Brekke, O. Holt, T. B. Jones, T. Robinson, A. Hedberg, B. Thidé, M. Crochet, and H. J. Lotz, Ionospheric modification experiments in northern Scandinavia, J. Atmos. Terr. Phys., 44, 1025, 1982.

Yeoman, T. K., D. M. Wright, T. R. Robinson, and M. T. Rietveld, High spatial and temporal resolution observations of an impulse-driven field line resonance in radar backscatter artificially generated with the Tromsø heater, Ann. Geophysicae, 15, 634, 1997.

Wright, D. M., and T. K. Yeoman, High resolution bistatic HF radar observations of ULF waves in artificially generated backscatter, Geophys. Res. Lett., 26, 2825, 1999. 